\title{
Commentary: Primary Transcripts of microRNAs Encode Regulatory Peptides
}

\author{
Shuo Lv, Lixia Pan and Guodong Wang * \\ Key Laboratory of Ministry of Education for Medicinal Plant Resource and Natural Pharmaceutical Chemistry, National \\ Engineering Laboratory for Resource Developing of Endangered Chinese Crude Drugs in Northwest of China, College of Life \\ Sciences, Shaanxi Normal University, Xi'an, China
}

Keywords: miRNA-encoded peptide (miPEP), pri-miRNA, long noncoding RNA (IncRNA), Arabidopsis, gene regulation

\section{A commentary on}

Primary Transcripts of microRNAs Encode Regulatory Peptides

by Lauressergues, D., Couzigou, J. M., Clemente, H. S., Martinez, Y., Dunand, C., Becard, G., et al. (2015). Nature 520, 90-93. doi: 10.1038/nature14346

\section{OPEN ACCESS}

Edited by:

Maria Eugenia Zanetti,

National University of La Plata,

Argentina

Reviewed by:

Richard Macknight,

University of Otago, New Zealand

Jean-philippe Combier,

Centre National de la Recherche

Scientifique, France

${ }^{*}$ Correspondence:

Guodong Wang

guodong_wang@snnu.edu.cn

Specialty section:

This article was submitted to Plant Genetics and Genomics,

a section of the journa

Frontiers in Plant Science

Received: 29 June 2016 Accepted: 08 September 2016 Published: 22 September 2016

Citation:

Lv S, Pan L and Wang G (2016) Commentary: Primary Transcripts of microRNAs Encode Regulatory

Peptides. Front. Plant Sci. 7:1436.

doi: 10.3389/fp/s.2016.01436
Noncoding RNAs (ncRNAs), including microRNAs (miRNAs) and long noncoding RNAs (lncRNAs), were assumed to be incapable of encoding proteins (Mercer et al., 2009; Rogers and Chen, 2013; Patil et al., 2014). miRNAs that derived from the primary miRNAs (pri-miRNAs) play crucial roles in post-transcriptional gene regulation by either repressing translation or guiding the degradation of complementary mRNA targets (Rogers and Chen, 2013). Transcriptome and high-throughput sequencing analyses have revealed a large number of ncRNAs in various organisms (Kapusta and Feschotte, 2014). It was found that ncRNAs were implicated in a variety of biological processes, including plant growth and development, and responses to environmental stresses (Mercer et al., 2009; Zhang et al., 2013; Kapusta and Feschotte, 2014).

Increasing evidence from both plants and animals has revealed that previously annotated IncRNAs have the capacity to encode small peptides (Ruiz-Orera et al., 2014; Lauressergues et al., 2015). In mammals, myoregulin (MLN), a lncRNA-encoded micropeptide, was reported to function in controlling muscle performance (Anderson et al., 2015). More specifically, MLN finely regulated calcium $\left(\mathrm{Ca}^{2+}\right)$ uptake through physical interaction with sarcoplasmic reticulum $\mathrm{Ca}^{2+}$ ATPase (SERCA). The removal of MLN in mice resulted in enhanced $\mathrm{Ca}^{2+}$ handling and improved exercise performance (Anderson et al., 2015). A second lncRNA-encoded peptide, termed dwarf open reading frame (DWORF), has been shown to enhance SERCA activity and $\mathrm{Ca}^{2+}$ load by displacing the SERCA inhibitors and mitigating their inhibitory activity (Nelson et al., 2016). Meanwhile, in plants $\sim 30,000$ ncRNAs have been identified with over 1700 transcripts designated as ncRNAs in Arabidopsis alone (Liu et al., 2015). The functionally characterized plant IncRNA-encoded peptides comprise ENOD40 (Early nodulin 40) that is required for plant-bacteria symbiotic interaction, IPS1 (Induced by phosphate starvation1) that is implicated in phosphate uptake, LDMAR (Long-day-specific male-fertility-associated RNA) that controls photoperiod-sensitive male sterility, and COOLAIR and COLDAIR that influence Arabidopsis flowering time by affecting FLC transcription (Zhang et al., 2013).

Pri-miRNAs have been recently reported to harbor short open reading frames (ORFs) that encode regulatory peptides, termed miRNA-encoded peptides (miPEPs), indicating that 
pri-miRNAs possess both protein-coding and non-coding roles (Lauressergues et al., 2015). The native expression of miPEPs could be detected using specific antibodies, and their expression patterns are the same as those of their corresponding miRNAs. Overexpression or exogenous application of two miPEPs, miPEP171b from Medicago truncatula and miPEP165a from Arabidopsis, enhanced the expression of their corresponding miRNAs, thereby potentiating the suppression of target genes involved in root development (Lauressergues et al., 2015). Collectively, this study revealed that miPEPs are functional peptides that could promote the accumulation of their associated pri-miRNAs and ultimately down-regulate target genes.

The identification of miPEPs is in line with increasing evidence that a large number of micropeptides were found to be encoded by previously unannotated short ORFs in lncRNAs (Ruiz-Orera et al., 2014; Lauressergues et al., 2015). An immediate question in future is to determine whether miPEPs exist in other organisms, and if so, how many of these miPEPs have a biological function? This further raises another question that with what approaches to detect and validate potential miPEPs. The existence of endogenous miPEPs have been experimentally demonstrated using immunoblot, GUS reporter analysis and overexpression studies for miPEP171b and miPEP165a (Lauressergues et al., 2015). The translation of pri-miR171b and pri-miR165a were also supported by ribosome profiling (Juntawong et al., 2014) although miPEP171b and miPEP165a have not been detected by mass spectrometry (Baerenfaller et al., 2008; Castellana et al., 2008). The identification of miPEPs by using computational prediction alone is challenging (Waterhouse and Hellens, 2015). As have been shown for the discovery of small ORFs (smORF)encoded peptides (Saghatelian and Couso, 2015), a combination of approaches including high-throughput RNA sequencing (RNA-seq), ribosome profiling, proteomics and bioinformatic is also required for identification of putative miPEPs (Aspden et al., 2014; Juntawong et al., 2014; Prabakaran et al., 2014).

A survey of fifty Arabidopsis pri-miRNAs revealed the presence of at least one putative smORF encoding a peptide in each sequence (Lauressergues et al., 2015). Further investigation of these putative miPEPs revealed that they did not share a common signature, suggesting that the regulatory activity of each putative miPEP is likely specific for their associated miRNA as have been experimentally shown for several miPEPs including miPEP171b and miPEP165a (Lauressergues et al., 2015). A key unanswered question will be how these different miPEPs perform their biological function, and whether the activation of primiRNA transcription is a prevalent mechanism for all miPEPs. The lncRNA-encoded micropeptides exert either inhibitory or stimulatory effects on their target genes in mammals (Anderson et al., 2015; Nelson et al., 2016). However, it remains unexplored whether miPEPs exert a negative effect on the expression of their associated miRNAs. Furthermore, it is intriguing to study whether any undiscovered components are involved in miPEP-mediated expression regulation, and whether any unknown means, which in turn modulate the positive effect of miPEPs.

As another aspect, miPEP synthesis and miRNA maturation occur in two physically distinct domains of pri-miRNAs. However, it is unclear how pri-miRNAs simultaneously coordinate their coding and non-coding capacities on the fact that cytoplasmic translation of pri-miRNA and nuclear maturation of miRNAs concurred. Regarding the coding function of pri-miRNA, genome editing to obtain lossof-function mutants, in addition to overexpression and exogenous application of synthetic peptides, is necessary to assess the function of miPEPs. It is known that many peptides, including CLE peptides, are subjected to post-translational modifications (Matsubayashi, 2011). However, whether miPEPs are post-translationally modified remains unclear. Additionally, considering their small size, it is of interest to investigate whether miPEPs are transported to mediate long distance signals similarly to that of those post-translationally modified peptides (Okamoto et al., 2013).

Because miPEPs specifically promote the transcription of their respective pri-miRNAs which result in down-regulation of target genes, they represent an efficient means for studying their corresponding miRNA families and improving yields in agronomical crops. Indeed, exogenous application of synthetic miPEP172c, which stimulates miR172c expression, eventually results in nodule formation in soybean (Couzigou et al., 2016). In this regard, miPEPs could be used as alternative tools to optimize agronomical traits of crops (Couzigou et al., 2015). However, one need aware that application of synthetic peptides in fields would be costly.

In conclusion, miPEPs identification highlights the dual function of pri-miRNAs which combine both protein-coding and non-coding capacities. Elucidating how miPEPs function will illuminate their important regulatory features and reveal an additional level of gene regulation.

\section{AUTHOR CONTRIBUTIONS}

GW and SL conceived and wrote the manuscript; LP critically reviewed the manuscript.

\section{ACKNOWLEDGMENTS}

We are grateful to Jinbin $\mathrm{Wu}$ (Wageningen University) for useful discussions. Research in our group is supported by the National Natural Science Foundation of China (31271575; 31200902), the Fundamental Research Funds for the Central Universities (GK201103005), the Specialized Research Fund for the Doctoral Program of Higher Education from the Ministry of Education of China (20120202120009), the Scientific Research Foundation for the Returned Overseas Chinese Scholars, State Education Ministry, and the Natural Science Basic Research Plan in Shaanxi Province of China (2014J M3064). 


\section{REFERENCES}

Anderson, D. M., Anderson, K. M., Chang, C. L., Makarewich, C. A., Nelson, B. R., McAnally, J. R., et al. (2015). A micropeptide encoded by a putative long noncoding RNA regulates muscle performance. Cell 160, 595-606. doi: 10.1016/j.cell.2015.01.009

Aspden, J. L., Eyre-Walker, Y. C., Phillips, R. J., Amin, U., Mumtaz, M. A., Brocard, M., et al. (2014). Extensive translation of small open reading frames revealed by Poly-Ribo-Seq. Elife 3:e03528. doi: 10.7554/eLife.03528

Baerenfaller, K., Grossmann, J., Grobei, M. A., Hull, R., Hirsch-Hoffmann, M., Yalovsky, S., et al. (2008). Genome-scale proteomics reveals Arabidopsis thaliana gene models and proteome dynamics. Nature 320, 938-941. doi: 10.1126/science.1157956

Castellana, N. E., Payne, S. H., Shen, Z., Stanke, M., Bafna, V., and Briggs, S.P. (2008). Discovery and revision of Arabidopsis genes by proteogenomics. Proc. Natl. Acad. Sci. U.S.A. 105, 21034-21038. doi: 10.1073/pnas.0811066106

Couzigou, J. M., André, O., Guillotin, B., Alexandre, M., and Combier, J. P. (2016). Use of microRNA-encoded peptide miPEP172c to stimulate nodulation in soybean. New Phytol. 211, 379-381. doi: 10.1111/nph.13991

Couzigou, J. M., Lauressergues, D., Bécard, G., and Combier, J. P. (2015). miRNAencoded peptides (miPEPs): a new tool to analyze the roles of miRNAs in plant biology. RNA Biol. 12, 1178-1180. doi: 10.1080/15476286.2015.1094601

Juntawong, P., Girke, T., Bazin, J., and Bailey-Serres, J. (2014). Translational dynamics revealed by genome-wide profiling of ribosome footprints in Arabidopsis. Proc. Natl. Acad. Sci. U.S.A. 111, E203-E212. doi: 10.1073/pnas.1317811111

Kapusta, A., and Feschotte, C. (2014). Volatile evolution of long noncoding RNA repertoires: mechanisms and biological implications. Trends Genet. 30, 439-452. doi: 10.1016/j.tig.2014.08.004

Lauressergues, D., Couzigou, J. M., Clemente, H. S., Martinez, Y., Dunand, C., Becard, G., et al. (2015). Primary transcripts of microRNAs encode regulatory peptides. Nature 520, 90-93. doi: 10.1038/nature14346

Liu, J., Wang, H., and Chua, N. H. (2015). Long noncoding RNA transcriptome of plants. Plant Biotechnol. J. 13, 319-328. doi: 10.1111/pbi.12336

Matsubayashi, Y. (2011). Small post-translationally modified peptide signals in Arabidopsis. Arabidopsis Book 9:e0150. doi: 10.1199/tab.0150

Mercer, T. R., Dinger, M. E., and Mattick, J. S. (2009). Long noncoding RNAs: insights into functions. Nat. Rev. Genet. 10, 155-159. doi: 10.1038/nrg2521
Nelson, B. R., Makarewich, C. A., Anderson, D. M., Winders, B. R., Troupes, C. D., Wu, F., et al. (2016). A peptide encoded by transcript annotated as long noncoding RNA enhances SERCA activity in muscle. Science 35, 271-275. doi: $10.1126 /$ science.aad4076

Okamoto, S., Shinohara, H., Mori, T., Matsubayashi, Y., and Kawaguchi, M. (2013). Root-derived CLE glycopeptides control nodulation by direct binding to HAR1 receptor kinase. Nat. Commun. 4, 2191. doi: 10.1038/ncomm s3191

Patil, V. S., Zhou, R., and Rana, T. M. (2014). Gene regulation by non-coding RNAs. Crit. Rev. Biochem. Mol. Biol. 49, 16-32. doi: 10.3109/10409238.2013.844092

Prabakaran, S., Hemberg, M., Chauhan, R., Winter, D., Tweedie-Cullen, R. Y., Dittrich, C., et al. (2014). Quantitative profiling of peptides from RNAs classified as noncoding. Nat. Commun. 5, 5429. doi: 10.1038/ncomms6429

Rogers, K., and Chen, X. (2013). Biogenesis, turnover, and mode of action of plant microRNAs. Plant Cell 25, 2383-2399. doi: 10.1105/tpc.113.113159

Ruiz-Orera, J., Messeguer, X., Subirana, J. A., and Alba, M. M. (2014). Long non-coding RNAs as a source of new peptides. Elife 3:e03523. doi: 10.7554/eLife.03523

Saghatelian, A., and Couso, J. P. (2015). Discovery and characterization of smORF-encoded bioactive polypeptides. Nat. Chem. Biol. 11, 909-916. doi: 10.1038/nchembio. 1964

Waterhouse, P. M., and Hellens, R. P. (2015). Coding in non-coding RNAs. Nature 520, 41-42. doi: 10.1038/nature 14378

Zhang, J., Mujahid, H., Hou, Y. X., Nallamilli, B. R., and Peng, Z. H. (2013). Plant long ncRNAs: a new frontier for gene regulatory control. Am. J. Plant Sci. 4, 1038-1045. doi: 10.4236/ajps.2013.45128

Conflict of Interest Statement: The authors declare that the research was conducted in the absence of any commercial or financial relationships that could be construed as a potential conflict of interest.

Copyright (c) 2016 Lv, Pan and Wang. This is an open-access article distributed under the terms of the Creative Commons Attribution License (CC BY). The use, distribution or reproduction in other forums is permitted, provided the original author(s) or licensor are credited and that the original publication in this journal is cited, in accordance with accepted academic practice. No use, distribution or reproduction is permitted which does not comply with these terms. 